Andreja Retelj

Oddelek za germanistiko z nederlandtistiko

in s skandinavistiko

Filozofska fakulteta Univerze v Ljubljani

andreja.retelj@ff.uni-lj.si
UDK 811.112.2'243:373.5(497.4)

DOI: $10.4312 /$ vestnik.8.217-233

\title{
SPRECHKOMPETENZ IM DAF-UNTERRICHT AN SLOWENISCHEN GYMNASIEN: VON DER THEORIE ZUR PRAXIS
}

Es liegt auf der Hand, dass Fremdsprachenkenntnisse aufgrund der wachsenden Globalisierung stets an Bedeutung gewinnen. Über eine hochentwickelte Fremdsprachenkompetenz zu verfügen, ist in der heutigen Gesellschaft fast zur Selbstverständlichkeit geworden. Durch die wachsenden wirtschaftlichen Kontakte gewinnt vor allem die mündliche Kommunikation immer mehr an Bedeutung und die Erwartungen an Fremdsprachenlernende, in verschiedenen Sprachsituationen mündlich schnell und angemessen kommunizieren zu können, nehmen aus diesem Grund ständig zu.

Slowenien hat aufgrund seiner geografischen Lage und der geschichtlichen Hintergründe sowie der engen wirtschaftlichen Kontakte bereits eine lange Unterrichtstradition in der deutschen Sprache. In den Grundschulen und Gymnasien wird heutzutage Deutsch überwiegend als zweite Fremdsprache gelernt. Schriftliche und mündliche Deutschkenntnisse sind am Arbeitsmarkt erwünscht und dies resultiert auch in einer Hochkonjunktur der Deutschkurse in den Sprachschulen während der letzten Jahre.

Unseres Wissens besteht keine aktuelle Studie über die Entwicklung der Sprechkompetenz im DaF-Unterricht, deswegen versteht sich dieser Beitrag als ein Versuch diese Lücke zu schließen. Zuerst wird dargestellt, wie die Sprechkompetenz im Gemeinsamen europäischen Referenzrahmen für Sprachen: lernen, lehren und beurteilen (weiter GeR) (Europarat 2001) und im Lehrplan für Deutsch für Gymnasien (2008), in den beiden für den slowenischen Kontext wichtigsten Dokumenten, definiert wird und welche Anregungen zur Förderung der Sprechkompetenz von Lernenden in diesen Dokumenten angeboten werden. Weiterhin werden die Resultate der Befragung der Lehrkräfte präsentiert, da die die Sprechkompetenz in der Praxis entwickeln. 


\section{DIE ENTWICKLUNG DER SPRECHKOMPETENZ IM GER}

Der GeR als etablierter Rahmen für den modernen Fremdsprachenunterricht beschreibt, „was Lernende zu tun lernen müssen, um eine Sprache für kommunikative Zwecke zu benutzen, und welche Kenntnisse und Fertigkeiten sie entwickeln müssen, um in der Lage zu sein, kommunikativ erfolgreich zu handeln« (Europarat 2001: 14). GeR unterscheidet schriftliche/mündliche Produktion (Schreiben, Sprechen, schriftliche/mündliche Interaktion, Rezeption (Hören, Lesen) und mündliche/schriftliche Sprachmittlung.

Laut dem GeR (Europarat 2001: 93) muss der Lernende beim Sprechen folgendes leisten:

- $\quad$ eine Mitteilung planen und organisieren können (kognitive Fertigkeiten);

- $\quad$ eine sprachliche Äußerung formulieren können (sprachliche Fertigkeiten);

- $\quad$ die Äußerung artikulieren können (phonetische Fertigkeiten).

Ähnliche Voraussetzungen liegen auch in dem bekanntesten Modell von Levelt (1989) vor, der von einer Konzeptualisierungs-, Formulierungs- und Artikulationsphase ausgeht.

Die Sprechkompetenz eines Lernenden zeigt sich, indem er verschiedene produktive mündliche Aktivitäten verwirklicht, z.B. der Lernende liest einen Text vor, fasst den Inhalt eines geschriebenen Textes zusammen, gibt Anweisungen, spricht spontan über ein Thema, hält ein Referat oder eine Präsentation, usw. Anhand dieser Sprechaktivitäten wird sofort klar, dass eine ganze Reihe von Faktoren Einfluss auf das Sprechen ausüben. Die mündliche Performanz eines Lernenden wird durch seine Aussprache bzw. Artikulation, seinen Wortschatzgebrauch, seine Grammatikbeherrschung, die Kohärenz des Gesagten, seine Sprech- und Sprach-Strategien, die Verwirklichung der Sprechabsicht usw. geprägt. All diese Faktoren, die beim Sprechen in Betracht gezogen werden müssen, machen es unmöglich, die Sprechkompetenz kurz und knapp zu beschreiben (vgl. Harsch 2007). Die Sprechkompetenz sollte demzufolge als Verflechtung von verschiedenen Teilkompetenzen angesehen werden.

Hinsichtlich des Sprechens kommt darum im GeR (Europarat 2001: 64-66) eine Reihe von globalen und spezifischen Skalen in Frage. Auf den Niveaus von A1 bis C2 werden die Erwartungen mit Deskriptoren bzw. Kann-Beschreibungen genau formuliert.

Das monologische Sprechen kann mithilfe von folgenden Skalen ${ }^{1}$ beschrieben werden: Mündliche Produktion allgemein; Zusammenhängendes monologisches Sprechen: Erfahrungen beschreiben; Zusammenhängendes monologisches Sprechen: Argumentieren; Öffentliche Ankündigungen/Durchsagen machen; Vor einem Publikum sprechen.

Weiterhin wird das Sprechen auch auf das dialogische Sprechen bzw. die mündliche Interaktion $^{2}$ bezogen, bei der sich der Sprachverwendende abwechselnd in der Rolle des Sprechers und des Hörers befindet. Für die mündliche Interaktion wurden 7 Skalen mit Deskriptoren ausgearbeitet: Mündliche Interaktion allgemein; Konversation; Informelle

1 Einzelne Skalen findet der interessierte Leser im GeR (Europarat 2001: 64-66).

2 Einzelne Skalen befinden sich im GeR (ibid: 78-85). 
Diskussion (unter Freunden); Formelle Diskussion und Besprechungen; Transaktionen: Dienstleistungsgespräche; Informationsaustausch; Interviewgespräche.

Eine weitere Rolle, die dem Sprechen im GeR zugeteilt wird, stellt die mündliche Sprachvermittlung dar, für die der GeR keine Deskriptoren enthält.

Mit einzelnen Skalen und deren Deskriptoren wird versucht die Entwicklung des Sprechens in der Fremdsprache auf Kompetenzstufen von A1 bis C2 genau und möglichst detailliert zu beschreiben. Obwohl die Anwendung der Deskriptoren eine transparente Einschätzung der Sprechkompetenz anbieten sollte, stellt sich in der Praxis heraus, dass es sich um ein äußerst komplexes Vorgehen handelt. Die Einstufung eines Lernenden auf ein bestimmtes, sich am GeR orientierenden Niveau setzt eine holistische Beurteilung seiner Sprechperformanz sowie die Beherrschung einzelner für das Sprechen relevanter Faktoren voraus. Zur Beurteilung der Sprechfertigkeit müssen neben den erwähnten Skalen für das Sprechen noch einige weitere spezifische Skalen aus dem GeR zur Hilfe genommen werden. Das Beurteilungsraster für die mündliche Sprachperformanz im GeR beinhaltet deshalb außerdem Deskriptoren für Spektrum, Korrektheit, Interaktion, Flüssigkeit und Kohärenz (ibid: 37-38).

Welcher Aktivitäten sich Lehrende zur Entwicklung der Sprechkompetenz ihrer Schüler im Einzelnen bedienen, ist im GeR nicht vorgeschrieben und wird der autonomen fachlichen Entscheidung der einzelnen Lehrkräfte überlassen, die sich auch an bestimmte curriculare Vorgaben zu halten haben.

\section{3 \\ DIE ENTWICKLUNG DER SPRECHKOMPETENZ IM LEHRPLAN FÜR DEUTSCH AN GYMNASIEN}

Die aktuellste Version des Lehrplans für Deutsch an Gymnasien ${ }^{3}$ wurde im Jahr 2008 veröffentlicht und ist für alle DaF-Lehrkräfte an slowenischen Schulen bindend. Schon am Anfang wird ausdrücklich betont, dass sich der Lehrplan am GeR orientiert und von den Definitionen der kommunikativen sprachlichen Kompetenz im GeR ausgeht (Holc et al. 2008: 5).

Im Lehrplan für Deutsch an Gymnasien (ibid: 11-12) wird der Entwicklung der Sprechkompetenz eine wichtige Rolle zugewiesen. Das Sprechen sollte im DaF-Unterricht als mündliche Produktion bzw. „Zusammenhängendes Sprechen“, mündliche Interaktion bzw. „An Gesprächen teilnehmen“ und mündliche Mediation bzw. Sprachmittlung gefördert werden.

Das erwartete Niveau der Sprechkompetenz von Lernenden wird in einzelnen Ausführungsmodulen ${ }^{4}$ festgelegt. An dieser Stelle werden nur die Ziele der Module II und III näher vorgestellt, die für Deutsch als zweite Fremdsprache gesetzt werden.

3 Učni načrt: Nemščina: Gimnazija: splošna, klasična, strokovna gimnazija: obvezni predmet in matura (420 ur), izbirni predmet (140 ur)

4 Eine detaillierte Beschreibung einzelner Module findet der interessierte Leser im Lehrplan für Deutsch (siehe Holc et al. 2008: 19-21). 
Modul II ist für Lernende mit Vorkenntnissen aus der Grundschule vorgesehen und wurde für 420 Schulstunden konzipiert. Für das erwartete Niveau B1-B2 nach dem Schulabschluss werden hinsichtlich des Sprechens folgende Ziele festgesetzt:

Die Lernenden können:

- $\quad$ schriftliche oder mündliche Texte mit allgemeiner Thematik mit eigenen Worten zusammenfassen und ihre Meinung dazu äußern,

- mit eigenen Texten etwas beschreiben, Inhalte zusammenfassen, planen, eigene Erfahrungen, Eindrücke und Gefühle äußern,

- an Gesprächen über allgemeine Themen teilnehmen und ihren eigenen Standpunkt zur Thematik kurz äußern,

- verschiedene Informationen weiterleiten,

- einzelne Wörter und Sätze verständlich aussprechen,

- ein ausgewähltes Thema mithilfe von $\mathrm{IKT}^{5}$ selbstständig präsentieren und darüber diskutieren (nach Holc et al. 2008: 19-20).

Am Ende des Moduls III (Anfänger, 420 Schulstunden, erwartetes Niveau B1) wird erwartet, dass die Lernenden mündlich:

- Inhalte eines Textes mit einfachen Worten wiedergeben,

- mit eigenen kurzen Texten etwas beschreiben, Inhalte zusammenfassen, planen, eigene Erfahrungen, Eindrücke und Gefühle, Zustimmen oder Ablehnen auf eine einfache Weise äußern,

- an Gesprächen, die sie persönlich interessieren, teilnehmen, wobei sie noch nicht so fließend sprechen und mehr Zeit brauchen,

- Informationen weiterleiten,

- einzelne Wörter und Sätze richtig aussprechen,

- kulturelle Unterschiede zwischen der eigenen Kultur und Kulturen im deutschsprachigen Raum wahrnehmen und sich dazu äußern (ibid).

Die erwarteten Ziele für das Sprechen auf den einzelnen Niveaus werden im Lehrplan auch in Form von Deskriptoren erfasst. Sie richten sich nach dem GeR und sind an die Lerngruppe „Jugendliche“ angepasst.

Laut dem Lehrplan (nach Holc et al. 2008: 24) wird erwartet, dass Lernende auf dem Niveau B1 und B2 bei der mündlichen Produktion/zusammenhängendem Sprechen folgendes leisten:

- B1: Der Schüler/Die Schülerin kann einfache Sätze bilden, anhand derer er/sie seine/ ihre Erfahrungen, Ereignisse, Träume, Wünsche und Ambitionen beschreiben kann. Er/Sie kann seine/ihre Anschauungen und Pläne kurz erläutern. Er/Sie kann eine

5 Informations- und Kommunikationstechnologie 
Geschichte erzählen oder den Inhalt eines Buches bzw. eines Films wiedergeben und seine/ihre Reaktionen darauf präsentieren.

- B2: Der Schüler/Die Schülerin kann klar und genau viele Sachverhalte aus verschiedenen für ihn/sie interessanten Bereichen beschreiben. Er/Sie kann seine/ihre Meinung zu bestimmten Problemen äußern und seine/ihre Einstellung begründen.

Für die mündliche Interaktion/an Gesprächen teilnehmen wird erwartet, dass Lernende auf $\mathrm{B} 1$ und $\mathrm{B} 2$ folgendes leisten:

- B1: Der Schüler/ Die Schülerin kann die meisten Sprachsituationen/sprachlichen Situationen auf Reisen, in denen die Zielsprache in bestimmten Zielgebieten gesprochen wird, bewältigen. Er/Sie kann auch unvorbereitet an Gesprächen teilnehmen, wenn es um für ihn/sie interessante oder alltägliche Themen geht (z.B. Familie, Hobbys, Arbeit, Reisen, aktuelle Ereignisse).

- B2: Der Schüler/Die Schülerin kann sich ziemlich fließend und spontan in der Fremdsprache äußern, sodass die Kommunikation ohne größere Schwierigkeiten verläuft. Er/Sie kann an Diskussionen über allgemeine Themen teilnehmen und mit Argumenten seine/ihre Standpunkte vertreten (ibid).

Laut dem Lehrplan für Deutsch an Gymnasien sollten Lernende am Ende des Gymnasiums das Niveau eines selbstständigen Sprachverwenders erreichen. Die Deskriptoren umfassen auf beiden dargestellten Niveaus die meisten sprachlichen Situationen aus dem Alltag der Lernenden und die meisten für sie relevanten Themen. Von den Lernenden wird erwartet, dass sie diese Situationen und Themen sprachlich erfolgreich bewältigen können. In Anlehnung an den GeR werden im Lehrplan folgende Themen vorgeschlagen: Persönliche Angaben; Familie und Wohnen; Schule und Ausbildung; Arbeit und Beruf; Dienstleistungen; Körper und Gesundheit; Freizeit und Unterhaltung; Reisen und Verkehr; Essen und Trinken, Einkaufen; Kultur, Kunst, Interkulturalität; zwischenmenschliche Beziehungen; Wissenschaft, Technologie, Massenmedien; Natur und Umweltschutz; Politik und Gesellschaft.

Für die Entwicklung der Sprechkompetenz werden im Lehrplan auch einige Aktivitäten empfohlen: lautes Vorlesen, Berichten mithilfe von Mitschriften, Beschreiben, Erzählen, Erklären, Rollenspiele, spontanes Sprechen, Singen, Erörtern, Zusammenfassen eines gesprochenen Textes in der Muttersprache oder Fremdsprache, Übersetzen (Holc et al. 2008: 11-12). Zur Förderung der Interaktion werden Dialoge, Rollenspiele und Simulationen empfohlen.

Im Kapitel 6.1.3 des Lehrplans werden die Entwicklung der Sprechkompetenz, die Rolle der Lehrkräfte dabei und die Richtlinien für die Unterrichtspraxis detaillierter beschrieben. Lehrende sollten die Entwicklung der Sprechkompetenz genau einplanen, sie sollten Lernmaterialien sorgsam vorbereiten und genaue Teilziele hinsichtlich dessen setzen, was Lernende durch bestimmte Sprechaktivitäten beim Unterricht oder 
selbstständige Arbeit erreichen sollten. Dabei sollten sie die Lernenden motivieren, unterstützen, im selbstständigen und autonomen Lernen fördern, für eine angenehme Lernatmosphäre sorgen, auf die Bedürfnisse einzelner Lernender eingehen, Aufgaben differenzieren und individualisieren.

Es werden einige Methoden und Techniken vorgeschlagen, mithilfe derer Lehrende die Sprechkompetenz ihrer Schüler entwickeln und fördern können,: z.B. geführte Dialoge, gemischte Reihenfolge in einem Dialog, Dialoge ergänzen, schematische Dialoge, funktionale Dialoge, Rollenspiele, Projektarbeit, Interviews, Fragebögen, Geschichten zusammenfassen, Sprechübungen mit Hilfsmitteln (Bilder, Videos, Fotos, Zeichnungen, Gegenstände, Stichwörter ...), Diskussionen (ibid: 34-35). Der Lehrende sollte den Unterricht so planen, dass die Lernenden genügend Lerngelegenheiten haben, um ihre Sprechkompetenz stufenweise zu entwickeln.

Die angezeigte Lehrerrolle, die Methoden und Techniken, die im Lehrplan zur Entwicklung und Förderung der Sprechkompetenz empfohlen werden, entsprechen dem Konzept des „Balanced Teaching“ (Thaler 2010), wobei die Lehrenden sich um eine Ausgewogenheit zwischen geschlossenen und offenen Unterrichtsmethoden bemühen, mittels derer sie die Lernenden zunächst mit den sprachlichen Mitteln vertraut machen und ihnen dann die Möglichkeit geben diese in möglichst authentischen Lernsituationen einzuüben und zu erlernen. Goh und Burns (2012: 151-152) schlagen eine Reihe von Sprechaktivitäten vor, mit denen Lehrende den Lernenden beim Sprechen helfen können.

In den beiden für slowenische DaF-Lehrende grundlegenden Dokumenten wird die Sprechkompetenz und ihre Entwicklung eher theoretisch dargestellt. Im Gegensatz zu den abstrakten und für beliebige Lernkontexte verfassten Beschreibungen im GeR findet man im Lehrplan für Deutsch an Gymnasien stellenweise auch einige Konkretisierungen für die Unterrichtspraxis. Diese werden meist als Ideen bzw. Anregungen hinsichtlich dessen angeboten, welche Methoden oder Techniken überhaupt eingesetzt werden können. Es hängt jedoch von den einzelnen Lehrkräften ab, ob sie diese überhaupt annehmen und in welchem Maße sie Lerngelegenheiten arrangieren.

\section{4}

\section{EMIPIRSCHER TEIL}

\subsection{Forschungsproblem}

Die wichtigsten Dokumente (der GeR und der Lehrplan für Deutsch an Gymnasien) setzen die Entwicklung der Sprechkompetenz als eines der wichtigsten Ziele des modernen Fremdsprachenunterrichts fest. Da die Richtlinien in beiden Dokumenten auf relativ abstrakte Weise verfasst werden, interessierte uns, wie die Umsetzung dieses Ziels in der Praxis erfolgt. Wir wollten herausfinden,

- welche Übungen Lehrende zur Förderung und Entwicklung der Sprechkompetenz einsetzen, 
- wie viel Zeit der Entwicklung der Sprechkompetenz im Unterricht gewidmet wird und

- welchen Einfluss der GeR auf die Unterrichtspraxis ausübt.

\subsection{Methode und Instrument}

Als Forschungsmethode bedienten wir uns der Befragung, weil wir eine größere Zahl von DaF-Lehrern und Lehrerinnen in die Studie einbeziehen wollten. Es wurde ein teilweise standardisierter Fragebogen mit 4 geschlossenen und 2 offenen Fragen erstellt. Zusätzlich wurden noch 2 Fragen zu demografischen Daten gestellt, und zwar zum Geschlecht und der Dauer der Berufserfahrungen. Für das Ausfüllen des Fragebogens waren 10 Minuten vorgesehen.

\subsection{Datenerhebung und Datenbearbeitung}

Der Fragebogen wurde am 20. August 2016 auf der jährlichen Deutschlehrerfortbildung in Ljubljana an Deutschlehrende verteilt. Von 65 verteilten Fragebögen im Papierformat erhielten wir 36 ausgefüllte Fragebögen zurück, die in die weitere Analyse einbezogen wurden. Zuerst wurden alle Daten in Excel übertragen und dann mithilfe der deskriptiven Statistik analysiert. Berechnet wurden Mittelwerte (MT), Standardabweichungen (STD) und Prozentzahlen (f\%).

\subsection{Teilnehmer}

An der Befragung nahmen 36 Deutschlehrerinnen aus verschiedenen Regionen Sloweniens teil. Obwohl die Stichprobe ( $\mathrm{N}=36)$ sehr klein ist und sich die Datenanalyse nicht einfach auf die Gesamtpopulation der Deutschlehrenden in Slowenien generalisieren lässt, bieten die Resultate trotzdem einen guten Einblick in den Schulalltag, vor allem, weil die Teilnehmerinnen zu der Gruppe gehören, die am häufigsten an Fortbildungen teilnehmen und bereit sind, Neues in ihrem Unterricht auszuprobieren.

Aus Tabelle 1 wird ersichtlich, dass die Teilnehmerinnen durchschnittlich 17,9 Jahre Deutsch als Fremdsprache unterrichten. Lehrerinnen mit solchen Berufserfahrungen sind schon so lange im Dienst, dass sie nicht mehr mit anfänglichen Schwierigkeiten zu kämpfen und ihren eigenen Lehrstil höchstwahrscheinlich schon entwickelt haben. Nur eine Teilnehmerin ist erst seit einem Jahr in der Schule tätig, eine weitere Teilnehmerin hat 30 Jahre Berufserfahrungen. 


\begin{tabular}{|c|c|c|c|c|}
\hline \multicolumn{5}{|c|}{ Wie lange unterrichten Sie schon Deutsch in der Schule? } \\
\hline N & MT & STD. & MIN & MAX \\
\hline 36 & 17.9 & 6.43 & 1 & 30 \\
\hline
\end{tabular}

Tabelle 1: Berufserfabrungen der Teilnebmerinnen

\subsection{Resultate und Diskussion}

Mit der ersten Frage wollten wir herausfinden, wie oft die Lehrenden ausgewählte Aktivitäten zur Förderung der Sprechfertigkeit in ihrem Unterricht einsetzen. Absichtlich wurden Aktivitäten aufgelistet, die entweder eine stark gelenkte oder eine etwas freiere mündliche Produktion ermöglichen sollten.

Aus der Tabelle 2 kann entnommen werden, dass die Lehrenden den Lernenden am häufigsten Fragen stellen (MT=4,3), ihnen Lückentexte geben, die sie ergänzen und dann vorlesen, $(\mathrm{MT}=3,9)$ oder sie lassen die Lernenden Rollenspiele zu bestimmten im Unterricht behandelten Themen vorbereiten $(\mathrm{MT}=3,6)$. Die Platzierung auf den ersten drei Stellen weist auf einen ziemlich stark gelenkten Unterricht hin, bei dem die Lehrenden alle Fäden in der Hand halten und eine größere Kontrolle über die Schüler und ihre Leistungen ausüben können, denn die ersten drei Aktivitäten setzen einen zweidimensionalen Kommunikationsweg voraus, der entweder im Plenum oder als Partnerarbeit realisiert werden kann.

Die Auswahl der häufigsten Sprechaktivitäten weist auch auf drei weitere wichtige Merkmale des DaF-Unterrichts hin: Das erste Merkmal ist der in der Unterrichtspraxis noch immer tief verankerte lehrerzentrierte Unterricht, bei dem die Lernenden eher passiv sind und nur auf die Impulse der Lehrkraft reagieren. Das zweite Merkmal deutet auf die Positionierung der Sprechfertigkeit hin, die auch als Reduktion auf das Vorlesen gesehen werden kann. Das Vorlesen der von den Schülern eingetragenen Lösungen kann nämlich eigentlich nur schwer oder gar nicht als Sprechübung, die zum freien Sprechen führt, bewertet werden. Vielmehr geht es bei solchen Übungen um das Üben der grammatischen Strukturen oder des Wortschatzes; das Sprechen spielt hier nur eine nebensächliche Rolle. Von der Tatsache, dass die grammatischen Kenntnisse eine wichtige Rolle für die slowenischen DaF-Lehrkräfte spielen, zeugt auch die Positionierung des Faktors „Richtigkeit“ bei der Bewertung der mündlichen Prüfung (siehe Tabelle 3). Das dritte Merkmal ist der starke Einfluss der Lehrwerke auf den DaF-Unterricht. Sowohl Lückentexte als auch Rollenspiele bzw. Dialoge sind Übungstypen, die in den Lehrwerken stark vertreten sind und im Unterricht demzufolge auch häufig eingesetzt werden. Aktivitäten, für welche die Lehrenden die Materialien selbst vorbereiten müssen, werden viel weniger eingesetzt, wie z.B. Simulationen, Debatten oder Diskussionen. Für solcherlei Aktivitäten muss auch mehr Zeit eingeplant werden als z.B. für einen fragend-entwickelnden Unterricht und die Steuerung des Lernprozesses ist außerdem geringer. Interessant ist die Rangierung auf den letzten zwei Plätzen, denn beide hier aufgelisteten Aktivitäten 
könnten die Kreativität fördern und auch andere nicht nur sprachliche Kompetenzen zur Geltung bringen, werden aber trotzdem ziemlich selten im Unterricht eingesetzt.

\begin{tabular}{|c|c|c|c|c|c|c|c|c|c|}
\hline & $\begin{array}{l}\text { Fr. } 1 \text { Wie oft setzten Sie } \\
\text { folgende Aktivitäten in Ihrem } \\
\text { Unterricht ein? }\end{array}$ & 1 & 2 & 3 & 4 & 5 & $\mathrm{~N}$ & MT & STD \\
\hline 1.6 & Frage-Antwort & $\begin{array}{c}0 \\
0 \%\end{array}$ & $\begin{array}{c}0 \\
0 \%\end{array}$ & $\begin{array}{c}3 \\
9 \%\end{array}$ & $\begin{array}{c}17 \\
49 \%\end{array}$ & $\begin{array}{c}15 \\
43 \%\end{array}$ & $\begin{array}{c}35 \\
100 \%\end{array}$ & 4.3 & 0.6 \\
\hline 1.5 & Ergänze die Lücken im Text! & $\begin{array}{c}0 \\
0 \%\end{array}$ & $\begin{array}{c}3 \\
9 \%\end{array}$ & $\begin{array}{c}9 \\
26 \%\end{array}$ & $\begin{array}{c}12 \\
34 \%\end{array}$ & $\begin{array}{c}11 \\
31 \%\end{array}$ & $\begin{array}{c}35 \\
100 \%\end{array}$ & 3.9 & 1.0 \\
\hline 1.7 & Rollenspiele/Dialoge & $\begin{array}{c}2 \\
6 \%\end{array}$ & $\begin{array}{c}2 \\
6 \%\end{array}$ & $\begin{array}{c}12 \\
33 \%\end{array}$ & $\begin{array}{c}13 \\
36 \%\end{array}$ & $\begin{array}{c}7 \\
19 \%\end{array}$ & $\begin{array}{c}36 \\
100 \%\end{array}$ & 3.6 & 1.1 \\
\hline 1.1 & $\begin{array}{l}\text { Mündliche Präsentation eines } \\
\text { Themas }\end{array}$ & $\begin{array}{c}1 \\
3 \%\end{array}$ & $\begin{array}{c}5 \\
14 \%\end{array}$ & $\begin{array}{c}11 \\
31 \%\end{array}$ & $\begin{array}{c}10 \\
29 \%\end{array}$ & $\begin{array}{c}8 \\
23 \%\end{array}$ & $\begin{array}{c}35 \\
100 \%\end{array}$ & 3.5 & 1.1 \\
\hline 1.3 & Beschreibe und ordne zu! & $\begin{array}{c}2 \\
6 \%\end{array}$ & $\begin{array}{c}9 \\
26 \%\end{array}$ & $\begin{array}{c}9 \\
26 \%\end{array}$ & $\begin{array}{c}12 \\
35 \%\end{array}$ & $\begin{array}{c}2 \\
6 \%\end{array}$ & $\begin{array}{c}34 \\
100 \%\end{array}$ & 3.1 & 1.1 \\
\hline 1.8 & Simulation & $\begin{array}{c}3 \\
9 \%\end{array}$ & $\begin{array}{c}8 \\
24 \%\end{array}$ & $\begin{array}{c}7 \\
21 \%\end{array}$ & $\begin{array}{c}15 \\
44 \%\end{array}$ & $\begin{array}{c}1 \\
3 \%\end{array}$ & $\begin{array}{c}34 \\
100 \%\end{array}$ & 3.1 & 1.1 \\
\hline 1.10 & Diskussion oder Debatte & $\begin{array}{c}2 \\
6 \%\end{array}$ & $\begin{array}{c}11 \\
31 \%\end{array}$ & $\begin{array}{c}9 \\
25 \%\end{array}$ & $\begin{array}{c}9 \\
25 \%\end{array}$ & $\begin{array}{c}5 \\
14 \%\end{array}$ & $\begin{array}{c}36 \\
100 \%\end{array}$ & 3.1 & 1.2 \\
\hline 1.11 & Story-Telling & $\begin{array}{c}4 \\
11 \%\end{array}$ & $\begin{array}{c}12 \\
33 \%\end{array}$ & $\begin{array}{c}8 \\
22 \%\end{array}$ & $\begin{array}{c}8 \\
22 \%\end{array}$ & $\begin{array}{c}4 \\
11 \%\end{array}$ & $\begin{array}{c}36 \\
100 \%\end{array}$ & 2.9 & 1.2 \\
\hline 1.9 & Problemlösung & $\begin{array}{c}6 \\
17 \%\end{array}$ & $\begin{array}{c}9 \\
26 \%\end{array}$ & $\begin{array}{c}14 \\
40 \%\end{array}$ & $\begin{array}{c}4 \\
11 \%\end{array}$ & $\begin{array}{c}2 \\
6 \%\end{array}$ & $\begin{array}{c}35 \\
100 \%\end{array}$ & 2.6 & 1.1 \\
\hline 1.4 & Beschreibe und zeichne nach! & $\begin{array}{c}7 \\
21 \%\end{array}$ & $\begin{array}{c}11 \\
33 \%\end{array}$ & $\begin{array}{c}10 \\
30 \%\end{array}$ & $\begin{array}{c}5 \\
15 \%\end{array}$ & $\begin{array}{c}0 \\
0 \%\end{array}$ & $\begin{array}{c}33 \\
100 \%\end{array}$ & 2.4 & 1.0 \\
\hline 1.2 & $\begin{array}{l}\text { Finde den Unterschied } \\
\text { (engl. Spot the difference) }\end{array}$ & $\begin{array}{c}10 \\
29 \%\end{array}$ & $\begin{array}{c}16 \\
46 \%\end{array}$ & $\begin{array}{c}7 \\
20 \%\end{array}$ & $\begin{array}{c}2 \\
6 \%\end{array}$ & $\begin{array}{c}0 \\
0 \%\end{array}$ & $\begin{array}{c}35 \\
100 \%\end{array}$ & 2.0 & 0.9 \\
\hline
\end{tabular}

Tabelle 2: Häufigkeit der Sprechaktivitäten im DaF-Unterricht

Darüber hinaus wollten wir herausfinden, welche Faktoren bei der mündlichen Befragung für die Lehrenden eine Rolle spielen. Bei der zweiten Frage konnten die Teilnehmer zwischen mehreren Antworten auswählen. Eine Rangierung war nicht vorgesehen. Die meisten Teilnehmer ( $\mathrm{f}=94 \%$ ) wählten den Faktor „Aussagekräftigkeit“ aus, was eigentlich erwartet worden war. Ein wenig überraschend ist die Platzierung des Faktors „Richtigkeit" ( $\mathrm{f}=78 \%$ ) an die die zweite Stelle, was darauf hindeutet, dass die Grammatik auch bei der mündlichen Befragung eine größere Rolle spielt als andere Faktoren, die bei der Bewertung der Sprechfertigkeit vielleicht relevanter wären. Da der DaF-Unterricht in Slowenien eine lange grammatikorientierte Tradition hat und grammatische Fehler eher als unerwünschte Erscheinung angesehen werden, schreiben die Teilnehmerinnen diesem Faktor noch immer große Bedeutung zu. Diese Antwort korreliert auch mit den am häufigsten eingesetzten Übungstypen, die vorher erwähnt wurden. 


\begin{tabular}{|c|l|c|c|}
\cline { 2 - 4 } \multicolumn{1}{l|}{} & Fr. 2 Welche Faktoren sind für Sie bei der mündlichen Befragung wichtig? & $\mathrm{F}$ & $\mathrm{f}(\%)$ \\
\hline 2.1 & Aussagekräftigkeit & 34 & 94 \\
\hline 2.4 & Richtigkeit & 28 & 78 \\
\hline 2.3 & Flüssigkeit & 27 & 75 \\
\hline 2.2 & Interaktion & 25 & 69 \\
\hline 2.5 & Aussprache & 25 & 69 \\
\hline
\end{tabular}

Tabelle 3: Wichtigkeit der Faktoren bei der mündlichen Bewertung

Im slowenischen Lehrplan für Deutsch als zweite Fremdsprache sind an Gymnasien 105 Stunden pro Schuljahr und insgesamt 420 Stunden für alle vier Jahre vorgeschrieben, was normalerweise in 3 Schulstunden pro Woche realisiert wird. Uns interessierte deswegen, wie viel Zeit die Lehrenden in den einzelnen Schulstunden für die Förderung der Sprechfertigkeit einplanen. Die meisten Teilnehmerinnen ( $\mathrm{f}=42 \%$ ) widmen den Sprechübungen zwischen 15 und 20 Minuten. Es folgen jene Teilnehmerinnen ( $f=36$ $\%$ ), die zwischen 5 und 15 Minuten Zeit für das Sprechen einplanen. In der Praxis heißt das, dass während einer Schulstunde bis zur Hälfte der Unterrichtszeit gesprochen wird. Das scheint auf den ersten Blick ziemlich viel zu sein. Wie aus der ersten Frage (siehe Tabelle 2) abzuleiten ist, kommen aber eher geschlossene Übungen vor, bei denen die Lernenden stark gelenkt werden oder das Gespräch verläuft nach dem Ping-Pong-Prinzip mit Fragen und Antworten zwischen Lehrenden und Lernenden. Das Ziel solcher Übungen ist wahrscheinlich bestimmte Strukturen und/oder Wortschatz zu trainieren, also das, was im Unterricht zurzeit gerade behandelt wird, auch mündlich einzuüben.

\begin{tabular}{|l|l|c|c|}
\hline Fr. 3 & $\begin{array}{l}\text { Wie viel Zeit widmen Sie den Sprechübungen in den einzelnen Schulstunden } \\
\text { (45 Minuten) }\end{array}$ & $\mathrm{F}$ & $\mathrm{f} \%$ \\
\hline 3.3 & Zwischen 15 und 20 Minuten & 15 & 42 \\
\hline 3.2 & Zwischen 5 und 15 Minuten & 13 & 36 \\
\hline 3.1 & Weniger als 5 Minuten & 7 & 19 \\
\hline 3.4 & Mehr als 20 Minuten & 1 & 3 \\
\hline Gesamt & 36 & 100 \\
\hline
\end{tabular}

Tabelle 4: Zeitlicher Rabmen für die Sprechaktivitäten

Nachdem die Lehrerinnen im Rahmen der Fortbildung die in der Tabelle 5 aufgelisteten Übungen zur Förderung der Sprechfertigkeit praktisch ausprobiert hatten, wurden sie nach der Nützlichkeit der Übungen für ihren Unterricht gefragt. Aus der Platzierung in der Tabelle 5 wird ersichtlich, dass die Übungen, die das freie Sprechen im Unterricht fördern, positiv bewertet wurden. Alle ausprobierten Aktivitäten wurden mit ziemlich hohen Mittelwerten bewertet. 
An die erste Stelle wurde die Methode „Speed-Dating“ platziert $(\mathrm{MT}=4,8)$. Da mit dieser Methode das Sprechen in größeren Gruppen gefördert werden kann, scheint diese Auswahl nicht überraschend. Viele Lehrerinnen empfinden die Sprechaktivitäten in großen Klassen als äußert problematisch (siehe Tabelle 6), weil man solche Lernsituationen nur schwer unter Kontrolle halten und die Lernenden auch nur schwer begleiten kann. In dieser Aktivität haben Lehrerinnen eine Möglichkeit dafür erkannt, wie sie ihre ganze Klasse zum Sprechen bringen und trotzdem eine mäßige Lenkung ausüben könnten. Den zweiten Platz besetzt die Aktivität „Frage-Antwort nach Mustersätzen“ (MT=4,6). Dieser Übungstyp ermöglicht eine ziemlich starke Steuerung des Lernprozesses, die Partnerarbeit lässt den Lehrenden einen gewissen Überblick über die Mitarbeit der Schüler und er kann auch in heterogenen Klassen eingesetzt werden. Bei einer so hohen Bewertung dieses Übungstyps könnte auch die Tatsache eine wichtige Rolle spielen, dass bereits viele vorgefertigte Materialien existieren und mit nur wenigen oder keinen Veränderungen sofort im Unterricht eingesetzt werden können. Dasselbe lässt sich für die drittplatzierte Aktivität „eine Bildergeschichte erzählen“ $(\mathrm{MT}=4,5)$ vermuten. Bei dem beschriebenen Workshop wurden etliche Lernmaterialien mit Bildergeschichten präsentiert, die den meisten Teilnehmerinnen zwar bekannt waren, aber dennoch nicht für Sprechaktivitäten verwendet wurden. Was möglicherweise zur hohen Bewertung dieser Aktivität beitrug, ist der Steuerungsgrad, der beliebig festgelegt werden kann. Darüber hinaus bewerteten die Lehrerinnen noch zwei weitere Sprechaktivitäten $(M T=4,2)$ sehr hoch, die vor allem für größere Gruppen in Frage kommen. Bei der Aktivität „Kartenaustausch“ wurde am Beispiel von Fragen zu einem Thema in der Praxis gezeigt, wie Lernende miteinander über ein Thema sprechen können und bei der Aktivität „Geschichten aus der Tasche“, wie eine Gruppe zum Sprechen angeregt werden kann. Interessanterweise wurde auch die Aktivität „Bilddiktat“ mit einem hohen Mittelwert bewertet $(\mathrm{MT}=4,1)$, obwohl gerade diese Aktivität, wie aus der Tabelle 2 (siehe Fr. 1.4) ersichtlich ist, eher selten eingesetzt wird. Wir glauben, dass Lehrerinnen diese Aktivität selber nicht wirklich sinnvoll einsetzen konnten und sich erst während des Workshops davon überzeugen konnten, dass sie wirkungsvoll und dazu sogar unterhaltsam ausfallen kann. Auch die beiden Präsentationsformen (Fr. 4.6 und 4.10) wurden als nützlich bewertet $(\mathrm{MT}=3,8)$. Obwohl das Präsentieren eines Themas schon jetzt häufig im Unterricht vorkommt (siehe Tabelle 2; F 1.1), könnten beide Präsentationstechniken auch im DaF-Unterricht Einsatz finden. Mit einem Durchschnittswert von 3,7 wurden zwei Aktivitäten bewertet: „Rory Cubes Geschichten“ und „Bilder kommentieren“. Beide Aktivitäten waren für die meisten Lehrerinnen neu. Mit dem Mittelwert 3,6 und damit auf dem letzten Platz wurde die Aktivität „Wimmelbilder sprechen lassen“ rangiert. Aus den Bewertungen der Lehrerinnen lässt sich schließen, dass alle Aktivitäten, die sie praktisch ausprobierten, großes Potenzial für die Sprechförderung im Unterricht in sich tragen. 


\begin{tabular}{|l|l|c|c|c|c|c|c|c|c|}
\hline \multirow{2}{*}{ Fr. 4} & Wie nützlich sind Ihrer Meinung nach folgende Sprechaktivitäten? \\
\cline { 2 - 10 } & Aktivität & 1 & 2 & 3 & 4 & 5 & $\mathrm{~N}$ & MT & STD \\
\hline 4.11 & Speed-Dating & 1 & 0 & 0 & 4 & 29 & 34 & 4.8 & 0.7 \\
& & $3 \%$ & $0 \%$ & $0 \%$ & $12 \%$ & $85 \%$ & $100 \%$ & & \\
\hline 4.1 & Frage-Antwort nach & 0 & 0 & 3 & 9 & 22 & 34 & 4.6 & 0.7 \\
& Mustersätzen & $0 \%$ & $0 \%$ & $9 \%$ & $26 \%$ & $65 \%$ & $100 \%$ & & \\
\hline 4.2 & eine Bildergeschichte & 0 & 0 & 5 & 7 & 23 & 35 & 4.5 & 0.7 \\
& erzählen & $0 \%$ & $0 \%$ & $14 \%$ & $20 \%$ & $66 \%$ & $100 \%$ & & \\
\hline 4.4 & Kartenaustausch & 2 & 0 & 4 & 8 & 17 & 31 & 4.2 & 1.1 \\
& & $6 \%$ & $0 \%$ & $13 \%$ & $26 \%$ & $55 \%$ & $100 \%$ & & \\
\hline 4.9 & Geschichten aus der & 1 & 1 & 6 & 11 & 17 & 36 & 4.2 & 1.0 \\
& Tasche & $3 \%$ & $3 \%$ & $17 \%$ & $31 \%$ & $47 \%$ & $100 \%$ & & \\
\hline 4.3 & Bilddiktat & 1 & 2 & 6 & 9 & 17 & 35 & 4.1 & 1.1 \\
& & $3 \%$ & $6 \%$ & $17 \%$ & $26 \%$ & $49 \%$ & $100 \%$ & & \\
\hline 4.6 & Pecha Kucha & 1 & 2 & 4 & 12 & 5 & 24 & 3.8 & 1.0 \\
& Präsentationstechnik & $4 \%$ & $8 \%$ & $17 \%$ & $50 \%$ & $21 \%$ & $100 \%$ & & \\
\hline 4.10 & Ted-X-Format & 1 & 0 & 8 & 13 & 5 & 27 & 3.8 & 0.9 \\
& & $4 \%$ & $0 \%$ & $30 \%$ & $48 \%$ & $19 \%$ & $100 \%$ & & \\
\hline 4.8 & Rory Cubes & 1 & 3 & 12 & 6 & 12 & 34 & 3.7 & 1.1 \\
& Geschichten erfinden & $3 \%$ & $9 \%$ & $35 \%$ & $18 \%$ & $35 \%$ & $100 \%$ & & \\
& und erzählen & & & & & & & & \\
\hline 4.7 & Bilder kommentieren & 1 & 2 & 5 & 12 & 4 & 24 & 3.7 & 1.0 \\
& & $4 \%$ & $8 \%$ & $21 \%$ & $50 \%$ & $17 \%$ & $100 \%$ & & \\
\hline \multirow{2}{*}{4.5} & Wimmelbilder & 2 & 4 & 5 & 10 & 7 & 28 & 3.6 & 1.2 \\
& sprechen lassen & $7 \%$ & $14 \%$ & $18 \%$ & $36 \%$ & $25 \%$ & $100 \%$ & & \\
\hline
\end{tabular}

Tabelle 5: Nützlichkeit der Sprechaktivitäten aus der Sicht der Lebrerinnen

Bei der fünften offenen Frage wollten wir herausfinden, mit welchen Schwierigkeiten sich die Lehrenden bei der Durchführung bestimmter Sprechaktivitäten im Unterricht konfrontiert sehen. Wir erhielten 32 Antworten, die in acht Kategorien eingeteilt wurden. Für die meisten Lehrerinnen ( $\mathrm{F}=18)$ stellt die Klassengröße das akuteste Problem dar. Viele berichten, dass sie Klassen mit bis zu 33 Lernenden unterrichten und darin den Grund dafür sehen, dass die Lernenden so nur wenige Gelegenheiten zur Teilnahme an einem Gespräch bekommen. Vor allem bei freien Sprechübungen ist das problematisch. Das zweitgrößte Problem für die Lehrerinnen $(\mathrm{F}=13)$ ist mit der Kontrolle über die Mitarbeit der Lernenden und der Korrektur verbunden. Wenn die Lernenden eine Sprechaktivität in Gruppen oder in Paaren ausüben, sehen sich die Lehrkräfte in ihrer Rolle eingeschränkt, weil sie die Äußerungen der Lernenden nicht korrigieren können. Als drittes Problem wird ein großer Leistungsunterschied genannt $(\mathrm{F}=8)$, denn die Lernenden sollen sehr unterschiedliche Vorkenntnisse haben, was mit einem hohen Aufwand bei der Aufgabenerstellung verbunden ist. In die vierte Kategorie „Zeitliche Beschränkung“ wurden 
alle Antworten eingeordnet, die das Problem der Dauer ansprechen. Freie Sprechübungen dauern länger und einige Lehrerinnen $(\mathrm{F}=7)$ meinen, man könne diese Zeit viel besser nutzen, wenn man den Unterricht selbst lenkt. Darüber hinaus berichten die Lehrerinnen $(\mathrm{F}=6)$, dass die Schüler unvorbereitet zum Unterricht kommen und bei den Sprechübungen deshalb nicht mitmachen können. Einen weiteren Hemmschuh für einen erfolgreichen Einsatz der Sprechübungen stellen auch persönliche Merkmale der Lernenden dar, wie z. B. Schüchternheit oder Unmotiviertheit $(\mathrm{F}=6)$. Einige Lehrerinnen $(\mathrm{F}=4)$ fühlen sich wegen des umfangreichen Lehrplans eingeschränkt und wieder andere $(F=4)$ empfinden Sprechübungen als problematisch, weil die Lernenden dann automatisch zu viel miteinander in der Muttersprache sprechen.

\begin{tabular}{|l|l|c|c|}
\hline Kategorie & $\begin{array}{l}\text { Fr. } 5 \text { Was sind für Sie die größten Schwierigkeiten bei der Förderung } \\
\text { der Sprechfertigkeit im Unterricht? }\end{array}$ & $\mathrm{F}$ & $\mathrm{f}$ \\
\hline K 1 & Zu große Klassen & 18 & 56 \\
\hline K 2 & Zu wenig Kontrolle & 13 & 40 \\
\hline K 3 & Zu großer Leistungsunterschied & 9 & 28 \\
\hline K 4 & Zeitliche Beschränkung & 7 & 22 \\
\hline K 5 & Unvorbereitete Schüler & 6 & 19 \\
\hline K 6 & Persönliche Merkmale der Lernenden & 6 & 19 \\
\hline K 7 & Zu umfangreicher Lehrplan & 4 & 12,5 \\
\hline K 8 & Gebrauch der Muttersprache & 4 & 12,5 \\
\hline
\end{tabular}

Tabelle 6: Schwierigkeiten bei der Förderung der Sprechfertigkeiten

Mit der letzten Frage sollte herausgefunden werden, inwiefern sich die Lehrenden bei der Entwicklung der Sprechfertigkeit des Gemeinsamen europäischen Referenzrahmens für Sprachen $(\mathrm{GeR})$ bedienen und wie hilfreich sie ihn finden. Auf diese Frage respondierten 31 Lehrerinnen. Ihre Antworten ließen sich in 3 Kategorien einteilen. Überraschenderweise wird der GeR hinsichtlich der Entwicklung der Sprechfertigkeit von vielen Befragten $(\mathrm{F}=15)$ überhaupt nicht angewendet. Einige Gründe dafür waren die Unübersichtlichkeit der Skalen (4-mal), zu allgemeine Deskriptoren (3-mal), kein praktischer Wert der Skalen (6-mal), ungeeignete Deskriptoren (1-mal) und auch die fehlende Vertrautheit mit den Deskriptoren (1-mal). Da der GeR im slowenischen Raum schon seit einigen Jahren bekannt ist und bei Lehrerfortbildungen mehrmals thematisiert wurde, hatten wir vermutet, dass Lehrende dieses Dokument viel häufiger zur Hand nehmen und die Deskriptoren bei ihrer praktischen Arbeit berücksichtigen. Für einige Lehrerinnen ( $\mathrm{F}=10)$ dient der GeR als Orientierungshilfe bei der Erstellung von Fragen für die mündliche Prüfung (4-mal), bei der Einschätzung des Schwierigkeitsgrades von verschiedenen Übungen (4-mal) und bei der jährlichen Planung (2-mal). Sofern sie in Lehrwerken vorkommen, finden die Deskriptoren als Kann-Beschreibung zur Selbstevaluation für Lernende ( $\mathrm{F}=6)$ Anwendung. 


\begin{tabular}{|l|l|c|c|}
\hline Kategorie & $\begin{array}{l}\text { Fr. } 6 \text { Wie hilfreich ist für Sie der Gemeinsame europäische } \\
\text { Referenzrahmen (GeR) im Hinblick auf die Entwicklung der } \\
\text { Sprechfertigkeit? }\end{array}$ & F $\%$ \\
\hline K 1 & Keine Anwendung & 15 & 48 \\
\hline K 2 & Orientation für Lehrende & 10 & 33 \\
\hline K 3 & Selbstevaluation für Lernende & 6 & 19 \\
\hline
\end{tabular}

Tabelle 7: Anwendung des GeR

Der GeR ist aus dem heutigen Fremdsprachenunterricht nicht mehr wegzudenken. Seit seiner Veröffentlichung fand er relativ schnell europa- und sogar weltweit Anerkennung und wurde als Grundlage für zahlreiche nationale Fremdsprachencurricula eingesetzt. Mit dem Ziel Transparenz und Vergleichbarkeit zu gewährleisten zeigen sich jetzt deutlich auch seine Desiderate. Gerade weil der GeR zur Abstraktion und Verallgemeinerung tendiert und damit für jeden Sprachkontext und jede Lernsituation geeignet ist, müssen Kompetenzskalen für jeden bestimmten Kontext und Bedarf, für alle Ziele und Lerngruppen konkretisiert und daran angepasst werden. Hinsichtlich des Sprechens bietet der GeR zahlreiche Skalen mit Deskriptoren, was jedoch für die Unterrichtspraxis im Schulalltag höchstwahrscheinlich zu abstrakt ist (vgl. Harsch 2007). Auch die Formulierungsweise der Deskriptoren kann dazu beitragen, dass man sich des GeR eher zur Feststellung der Sprechkompetenz als zur Planung der Aktivitäten für deren Entwicklung bedient.

Der Lehrplan für Deutsch orientiert sich am GeR und passt sich an eine bestimmte Lerngruppe und einen bestimmten Lernkontext an: die Jugendlichen an slowenischen Gymnasien. Im Unterschied zum GeR müssen sich die Lehrenden daran halten und die dort beschriebenen Prinzipien verwirklichen sowie die gesetzten Ziele erreichen. Im Lehrplan gibt es zwar einige Anregungen zur Entwicklung der Sprechkompetenz, die von den Lehrenden berücksichtigt werden können oder auch nicht. Was in der Klasse zur Förderung der Sprechkompetenz unternommen wird, bleibt weitgehend der autonomen Entscheidung der Lehrkräfte überlassen. Der Lehrplan setzt insofern nur das Niveau voraus, das am Ende eines Moduls erreicht werden sollte. Wie genau das verwirklicht werden sollte, lässt sich anhand des Lehrplans nicht feststellen. Die Resultate der Befragung der Lehrkräfte zeigen, dass das Sprechen vor allem auf Reproduktion reduziert wird. Häufig konzentriert sich das Sprechen im DaF-Unterricht auf das Beantworten der Fragen des Lehrers. Oftmals werden Sprechaktivitäten eingesetzt, die den Lernenden dabei helfen, ihren Wortschatz und grammatische Strukturen mündlich zu üben oder bestimmte sprachliche Mittel nach einem Muster anzuwenden. Es werden vor allem stark gesteuerte Übungsformate bevorzugt, die den Lehrkräften ermöglichen alle Fäden in der Hand zu halten. Mit solchen Übungen können sich Lernende nur schwer zu selbständigen 
Sprechern entwickeln (vgl. Schreiter 2001; Pauels 2007). Lehrende planen solche Aktivitäten, die das freie Sprechen fördern, relativ selten in den Unterricht ein, Wir glauben, dass die Ursache dafür auch darin liegt, dass die Lehrwerke, die im DaF-Unterricht eingesetzt werden und einen starken Einfluss auf die Unterrichtsplanung und -durchführung haben (Retelj 2014), solche Übungen nur spärlich enthalten. Interessanterweise wurden die meisten Sprechaktivitäten, die den Lehrenden im Workshop präsentiert wurden und die sie ausprobieren konnten, als positiv und nützlich bewertet. Die Vermutung liegt darum nahe, dass neben Zeitmangel und anderen Problemen, die beim Einsatz von Sprechaktivitäten in der Klasse angeführt wurden, auch ein eher bescheidenes Repertoire an Ideen ein Grund für den jetzigen Stand sein könnte. Es wird darum an die Organisatoren der Lehrerfortbildungen appelliert, den Lehrenden möglichst viele Unterrichtsmethoden zu präsentieren, sie erproben und danach darüber reflektieren zu lassen, auf welche Weise und in welchen konkreten Unterrichtssituationen diese Methoden eingesetzt werden könnten. Es hat sich nämlich deutlich gezeigt, dass nicht viele Lehrkräfte dazu in der Lage sind, den theoretischen Rahmen des GeR sowie des Lehrplans erfolgreich in die Praxis umzusetzen. Um slowenischen Gymnasiasten die Chance zur Entwicklung einer hohen Sprechkompetenz zu geben, müssten Lehrende einsehen und sich eingestehen, dass Übungen zur Förderung des Sprechens zwar zeitraubend sind, jedoch keinesfalls einen Zeitverlust darstellen.

\section{LITERATUR}

EUROPARAT (Hrsg.) (2001) Gemeinsamer europäischer Referenzrahmen für Sprachen: lernen, lehren, beurteilen Berlin/München: Langenscheidt.

GOH, Christine C. M./Anne BURNS (2012) Teaching speaking: A holistic approach. New York: Cambridge University Press.

HARSCH, Claudia (2007) Der gemeinsame europäische Referenzrahmen für Sprachen: Leistungen und Grenzen. Saarbrücken: Vdm Verlag Dr. Müller.

HOLC Nada et al. (2008) Učni načrt: Nemščina: Gimnazija: splošna, klasična, strokovna gimnazija: obvezni predmet in matura (420 ur), izbirni predmet (140 ur) http:// eportal.mss.edus.si/msswww/programi2010/programi/media/pdf/un_gimnazija/ un_nemscina_gimn.pdf

LEVELT, Willem. J. M. (1989) Speaking: From intention to articulation. Cambridge: MIT Press.

PAUELS, Wolfgang (2007) Kommunikative Übungen. In: Bausch, Karl-Richard \& Herbert Christ \& Hans-Jürgen Krumm (Hrsg.), Handbuch Fremdsprachenunterricht. Tübingen: Francke, 302-305.

RETELJ, Andreja (2014) Pouk nemščine v gimnazijah skozi prizmo metod in pristopov k poučevanju. Vestnik za tuje jezike, 6/1, 221-234. 
SCHREITER, Ina (2001) Mündliche Sprachproduktion. In: Helbig, Gerhard \& Lutz Götze \& Henrici Gert \& Hans-Jürgen Krumm (Hrsg.), Deutsch als Fremdsprache. Ein internationales Handbuch. 2. Halbbd. Berlin: de Gruyter, 908-920.

THALER, Engelbert (2010) Lernerfolg durch Balanced Teaching. Berlin: Cornelsen Verlag.

\section{POVZETEK}

\section{Govorna zmožnost pri pouku nemščine v slovenskih gimnazijah: od teorije do prakse}

V članku predstavljamo, kako se razvija govorna zmožnosti pri pouku nemščine v slovenskih gimnazijah. Vključili smo tri vidike: opredelitev govorne zmožnosti v Skupnem evropskem referenčnem okviru (kratko SEJO), Učni načrt za nemščino za gimnazije in učne dejavnosti za poučevanje in razvijanje govorne zmožnosti pri pouku. Analiza obeh dokumentov kaže, da se govorni zmožnosti pripisuje velik pomen. V obeh dokumentih najdemo smernice, kaj se od učenca na posamezni ravni pričakuje in kakšno jezikovno znanje in kompetence mora učenec pokazati, da se ga lahko umesti na določeno raven. Učni načrt smernice v SEJO še dodatno prilagodi učnemu kontekstu in cilji skupini. Od slovenskega gimnazijca se ob koncu šolanja tako pričakuje raven samostojnega govorca. Oba dokumenta teoretsko opredelita govorno zmožnost, vendar ne dajeta vpogleda, kako se govorna zmožnost razvija v posameznih razredih. Analiza ankete med učitelji je pokazala, da se govorna zmožnost razvija le v omejenih okvirih. Kot najpogostejši vzrok za to učitelji navajajo premalo časa, težavno popravljanje napak, premajhno kontrolo nad razredom, slabo znanje učencev, nemotiviranost in preveliko število učencev v razredu. Prav tako je iz ankete razvidno, da učitelji za razvijanje govorne zmožnosti izbirajo le majhen repertoar učnih dejavnosti. Da bi slovenski gimnazijci resnično lahko razvili visoko govorno zmožnost, bi morali učitelji temeljito spremeniti pogled na svojo in učenčevo vlogo v razredu, v pouk vpeljevati tudi učne dejavnosti, ki razvijajo prosti govor, predvsem pa spodbujati učenčevo samostojnost in odgovornost za lastno učenje.

Ključne besede: govorna zmožnost, poučevanje govorne zmožnosti, učne dejavnosti, nemščina kot tuji jezik, učni načrt, SEJO

\section{ABSTRACT}

\section{Speaking Competence in German as a Foreign Language Class in Slovenian Secondary Schools: From Theory to Practice}

This article presents the speaking competence in German language classes in Slovenian secondary schools from three different perspectives: it gives the definition of speaking competence within the 
Common European Framework of Reference for Languages (CEFR), within the Syllabus for German for Secondary Schools, and within learning activities for teaching and developing speaking competence in German foreign language classes. The analysis of both documents shows that great importance is attributed to speaking competence, but, at the same time, both documents provide us with guidelines in terms of what is expected from each student at a certain CEFR level and what kind of language competence and knowledge a student is required to demonstrate in order to qualify for a certain level. The syllabus additionally adapts CEFR guidelines to the learning context and target groups. It is thus expected of a Slovenian secondary school student to be able to communicate on an independent user level at the end of the secondary school education. Both documents define speaking competence theoretically. However, the documents do not provide insight into the development of speaking competence in particular classes. The analysis of the survey conducted among teachers showed that speaking competence is developed only within limited frameworks. The most frequent reasons were lack of time, difficult error correction, insufficient classroom control, poor students' performance, lack of motivation and large classes. The survey also revealed that teachers chose only a small repertoire of learning activities for developing speaking competence. In order for Slovenian secondary students to truly develop high-level speaking competence, teachers should considerably alter their views on their own role, as well as a student's role in the classroom, introduce learning activities into language classes which develop free speech, and above all encourage students' independence and responsibility for their own learning process.

Keywords: speaking competence, teaching speaking, CEFR, speaking activities, Syllabus, German as a foreign language 